\title{
Towards accurate component properties of the Hyades binary $\theta^{2}$ Tau
}

\author{
P. Lampens, Y. Frémat, P. De Cat and H. Hensberge \\ Koninklijke Sterrenwacht van België, Ringlaan 3, 1180 Brussel, Belgium
}

\begin{abstract}
$\theta^{2}$ Tau is a well-detached, "single-lined" Hyades binary consisting of two midtype A stars which both lie in the lower Cepheid instability strip. As a matter of fact, component $A$ is a classical $\delta$ Scuti star showing a complex pattern of pulsations while component B might also be a $\delta$ Scuti pulsator (Breger et al. 2002). We acquired new high-resolution, high signal-to-noise spectra in order to determine accurate properties for the components of this system. Combining both spectroscopy and long-baseline optical interferometry, we were able to derive the orbital parallax and the component masses with unprecedented accuracy. Such constraints on the physical properties of the components are needed for a deep understanding of the pulsation physics. We also believe that $\theta^{2} \mathrm{Tau}$ is an appropriate target to explore, in an empirical way, the possible interaction(s) between pulsation on the one hand and rotation and binarity on the other hand.
\end{abstract}

\section{Introduction}

Our research currently focuses on binary and multiple stars with at least one pulsating component. In some cases, both the theories of stellar evolution and of pulsation can be tested and refined. Accurately derived component properties compared to suitably chosen theoretical isochrones allow to obtain information on the object's age and evolutionary status and to help discriminate among various possible pulsation models. We selected the $\delta$ Scuti star $\theta^{2}$ Tau for a

\footnotetext{
${ }^{1}$ Short research note based on a poster presented at IAU Symp. 240 "Binaries as Critical Tools and Tests in Contemporary Astrophysics", Prague, Aug. 2006

${ }^{2}$ Based on OHP (Observatoire de Haute-Provence) observations and spectral data retrieved from the ELODIE archive (http://atlas.obs-hp.fr/elodie/).
} 
detailed study because a) it is a well-detached, spectroscopic binary resolved by long-baseline interferometry (Armstrong et al. 2006) it is a member of the Hyades open cluster at a mean distance of 45 pc (Perryman et al. 1998) the evolutionary status of its components is still under debate (Torres et al. 1997 (TSL97); Lastennet et al. 1999; Armstrong et al. 2006).

\section{Recent spectroscopic campaign: spectra and analysis}

High-resolution spectra were acquired with the ELODIE Echelle spectrograph at the 1.93-m telescope of the Observatoire de Haute-Provence (OHP, France) through regular service mode observations from March 2005 until March 2006. These spectra were obtained over the full wavelength range (including the hydrogen lines) with a typical S/N of 100 and cover an entire orbital cycle. We also made use of some older observations from the ELODIE data base (Moultaka et al. 2004) as well as of the spectra acquired by TSL97 during the years 1989 till 1996 at the Oak Ridge Observatory (Harvard, Massachusetts).

Since the spectral lines of both components never separate completely due to the Doppler shifts being smaller than the line widths, we applied the spectra disentangling technique using the code KOREL developed by Hadrava (1995). For a description of the technique and usage, we respectively refer to Hadrava (2004) and Hensberge \& Pavlovski (2007). Studied spectral regions were selected for their high intrinsic content of radial velocity information (Verschueren \& David 1999, Hensberge et al. 2000). Application of KOREL to the above mentioned spectra enabled us to extract the individual contribution of each component to the observed spectra together with the orbital parameters, and therefore also to produce for each component of the binary a set of 117 KOREL radial velocities relative to the systemic velocity with a homogeneous coverage in amplitude and in orbital phase.

\section{Combined orbital analysis}

We next combined the previous data set with 34 best-fit angular separations $(\rho)$ and position angles $(\theta)$ as derived from the interferometric measurements (Armstrong et al. 2006). An astrometric-spectroscopic orbit was computed using the VBSB2 code which performs a global exploration of the parameter space followed by a simultaneous least-squares minimization (Pourbaix 1998). The combination of these measurement techniques is a powerful tool for obtaining accurate fundamental parameters. The resulting orbital elements and standard deviations of the best orbital solution in the sense of minimum least-squares residuals are presented in Table 1 . The previous results of a similar computation performed by Torres et al. (1997) are also listed for comparison. While there is 
Table 1: Orbital elements with standard deviations including orbital parallax and dynamical masses.

\begin{tabular}{lcc}
\hline Orbital element & This work & TSL97 results \\
\hline $\mathrm{P}($ days $)$ & $140.7285 \pm 0.0004$ & $140.7282 \pm 0.0009$ \\
$\mathrm{~T}$ & $1990.7630 \pm 0.0002$ & $1993.0752 \pm 0.0008$ \\
$\mathrm{e}$ & $0.7353 \pm 0.0004$ & $0.727 \pm 0.005$ \\
$\mathrm{a}(")$ & $0.0188 \pm 0.0001$ & $0.0186 \pm 0.0002$ \\
$\mathrm{i}\left(^{\circ}\right)$ & $47.65 \pm 0.12$ & $46.2 \pm 1.0$ \\
$\Omega\left(^{\circ}\right)$ & $354.59 \pm 0.12$ & $171.2 \pm 1.8$ \\
$\omega\left(^{\circ}\right)$ & $234.61 \pm 0.12$ & $236.4 \pm 1.1$ \\
$\mathrm{~V}_{0}(\mathrm{~km} / \mathrm{s})$ & - & $+39.5 \pm 0.2$ \\
$\kappa=\frac{M_{\mathrm{B}}}{M_{\mathrm{A}}+M_{\mathrm{B}}}$ & $0.452 \pm 0.002$ & $0.46 \pm 0.05$ \\
$\pi_{d y n}(\mathrm{mas})$ & $21.20 \pm 0.13$ & $21.22 \pm 0.76$ \\
$\mathrm{~A}(\mathrm{~A} . \mathrm{U})$. & $0.8879 \pm 0.0005$ & $0.88 \pm 0.04$ \\
mass A $\left(M_{\odot}\right)$ & $2.58 \pm 0.04$ & $2.4 \pm 0.3$ \\
mass B $\left(M_{\odot}\right)$ & $2.13 \pm 0.02$ & $2.1 \pm 0.2$ \\
$\mathrm{~K} 1(\mathrm{~km} / \mathrm{s})$ & $33.86 \pm 0.11$ & $33.18 \pm 0.49$ \\
$\mathrm{~K} 2(\mathrm{~km} / \mathrm{s})$ & $40.98 \pm 0.21$ & $38 \pm 2$ \\
\hline System mass $\left(M_{\odot}\right)$ & $4.71 \pm 0.10(2.1 \%)$ & $4.54 \pm 0.51(11.2 \%)$ \\
Time span $(\mathrm{yr})$ & 16.5 & 6.3 \\
\hline
\end{tabular}

a good agreement for most orbital parameters, the most conspicuous difference is the larger radial velocity amplitude of component B. In particular, note the improvement in accuracy of the orbital parallax as well as on the dynamical component masses. Using this parallax together with $V=3.40 \pm 0.03 \mathrm{mag}$ (Mermilliod et al. 1997) and $\Delta \mathrm{m}=1.12 \pm 0.03 \mathrm{mag}$ (Armstrong et al. 2006), we further derive the component absolute magnitudes $\mathrm{Mv}_{A}=0.36 \pm 0.04 \mathrm{mag}$ and $\mathrm{Mv}_{B}=1.48 \pm 0.04 \mathrm{mag}$.

\section{Cluster membership and future work}

Both components of $\theta^{2}$ Tau are among the more massive stars of the Hyades. They are located in the turnoff region of its colour-magnitude diagram. Provided that their physical properties are accurately known, both stars are useful indicators of chemical composition as well as age in this region (through fitting of theoretical isochrones). They also should allow to verify whether or not convective core overshooting occurs (Lebreton et al. 2001), as illustrated by the 


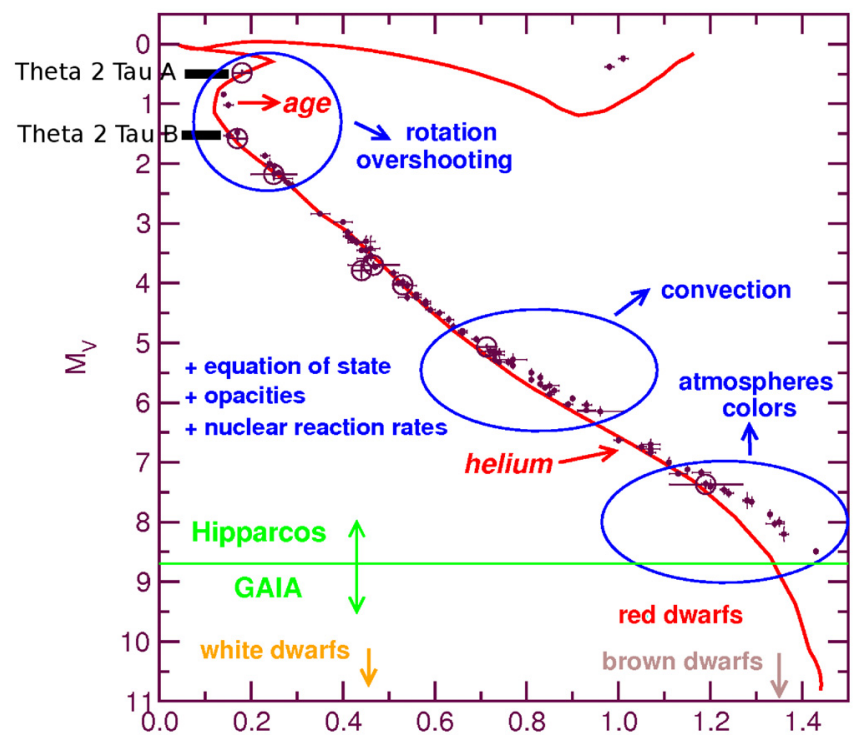

B-V

Figure 1: Hyades colour-magnitude diagram with an isochrone, $650 \mathrm{Myr}$ age, $\mathrm{Y}=0.26$, $[\mathrm{Fe} / \mathrm{H}]=+0.14$. and the locus of the components of $\theta^{2}$ Tau. (Courtesy of $\mathrm{Y}$. Lebreton, GAIA Information Sheets)

Hyades colour-magnitude diagram in Fig. 1.

Furthermore, Breger et al. ( 2002) proposed that both components might be $\delta$ Scuti pulsators. Therefore, the knowledge of accurate fundamental component properties holds great potential for a reliable pulsation modelling of each star. Further work will consist in using the disentangled component spectra to perform a detailed chemical analysis, to determine as accurately as possible the physical properties and evolutionary status and to carefully test whether or not convective overshooting is needed in the models. If more high-resolution spectra could be obtained with a much higher temporal resolution, we also would be able to study the pulsation characteristics in the line profiles of $\theta^{2}$ Tau $A$ and $\theta^{2}$ Tau B. 
Acknowledgments. We acknowledge funding from the OPTICON Transnational Access Programme for spectra collected with ELODIE (OHP). We thank P. Hadrava and D. Pourbaix for supplying the codes KOREL and VBSB2 respectively. We further thank G.Torres for providing us the Oak Ridge observations. Financial support from the Belgian Federal Science Policy is gratefully acknowledged (projects IAP P5/36 and MO/33/018).

\section{References}

Armstrong, J.T., et al. 2006, AJ, 131, 2643

Breger, M., et al. 2002, MNRAS, 336, 249

Hadrava, P. 1995, AAS 114, 393

Hadrava, P. 2004, in: "Spectroscopically and Spatially Resolving the Components of Close Binary Stars", Hilditch, R.W., Hensberge, H., \& Pavlovski, K. (eds.), ASP

Conf. Ser., 318, 86

Hensberge, H., Pavlovski, K., \& Verschueren, W. 2000, A\&A, 358, 553

Hensberge, H., \& Pavlovski, K. 2007, Proc. of IAU Symp. 240 "Binaries as Critical

Tools and Tests in Contemporary Astrophysics", Prague, Aug. 2006, in press

Lastennet, E., et al. 1999, A\&A, 349, 485

Lebreton, Y., et al. 2001, A\&A, 374, 540

Mermilliod J.C., Hauck B., \& Mermilliod M. 1997, A\&AS 124, 349

http://obswww.unige.ch/gcpd/gcpd.html

Moultaka, J., et al. 2004, PASP, 116, 693

Perryman, M., et al. 1998, A\&A, 331, 81

Pourbaix, D. 1998, AAS, 131, 377

Torres, G., et al. 1997, ApJ, 485, 167

Verschueren, W., \& David, M. 1999, A\&AS, 136,591 